\title{
Two steps forward, one step back: the pleiotropic effects of favoured alleles
}

\author{
Sarah P. Otto \\ Department of Zoology, University of British Columbia, Vancouver, British Columbia, V6T 1Z4, Canada \\ (otto@zoology.ubc.ca)
}

\begin{abstract}
Pleiotropy is one of the most commonly observed attributes of genes. Yet the extent and influence of pleiotropy have been underexplored in population genetics models. In this paper, I quantify the extent to which pleiotropy inhibits the spread of alleles in response to directional selection on a focal trait. Under the assumption that pleiotropic effects are extensive and deleterious, the fraction of alleles that are beneficial overall is severely limited by pleiotropy and rises nearly linearly with the strength of directional selection on the focal trait. Over a broad class of distribution of pleiotropic effects, the mean selective effect of those alleles that are beneficial overall is halved, on average, by pleiotropy. The fraction of new mutant alleles that are beneficial overall and that succeed in fixing within a population is even more severely limited when directional selection is weak, but it rises quadratically with the strength of directional selection. Finally, the mean selective effect of mutant alleles that are beneficial and succeed in fixing is reduced by one-third, on average, by pleiotropy. These results help to shape our understanding of the evolutionary inertia caused by pleiotropy.
\end{abstract}

Keywords: pleiotropy; adaptation; fixation probability; distribution of selective effects

\section{INTRODUCTION}

The available evidence indicates that pleiotropy is virtually universal.

(Wright 1968, p. 61)

Evolutionary biologists have long recognized that single genes affect multiple traits (Dobzhansky \& Holz 1943; Caspari 1952; Wright 1968), a phenomenon known as pleiotropy. Quantitative genetics models have embraced pleiotropy to help explain the covariance patterns among multiple traits (Lande 1980; Lande \& Arnold 1983; Turelli 1985; Slatkin \& Frank 1990), the maintenance of variation (Bulmer 1973; Gillespie 1984; Hill \& Keightley 1988; Barton 1990; Slatkin \& Frank 1990; Keightley \& Hill 1990), and the form of fitness landscapes (Wright 1969; Taylor \& Higgs 2000). By contrast, pleiotropy has been largely ignored in traditional population genetics models. Population genetics models describe the dynamics of particular alleles and tend to focus on the total fitness effect of an allele, ignoring how selection acts on the various traits altered by the allele. As a consequence, population genetics models typically ignore the many consequences of pleiotropy, which include limiting the rate of adaptation, altering the direction of evolutionary change over phenotypic space, and reducing the level of adaptation for other traits in response to direct selection on a trait.

The major obstacle for incorporating pleiotropy into population genetics models is our ignorance of how an allele affects multiple traits. A model introduced by Fisher (1930) has been resurrected recently to account for pleiotopy on the process of adaptation (Orr 1998). In the model of Fisher (1930), evolution occurs in $n$-dimensions corresponding to $n$ orthogonal traits under selection. Any particular mutation affects all of these traits to some degree (represented as a mutational vector pointing in a random direction), meaning that a special form of pleiotropy is explicitly included. Orr (1998) traced the series of mutations that bring a population towards a fitness optimum, allowing him to describe the distribution of selective effects that are likely to be seen among mutations fixed during the process of adaptation. The extent to which these results are dependent on the specific symmetry assumptions inherent in the model of Fisher (1930; mutations are equally likely to occur in any direction and the magnitude of mutations is independent of the current genotype or the angle of the mutation) is unknown. In this work, I use a simple alternative framework to explore the average effect of pleiotropy when populations respond to directional selection on one or a few focal traits.

The proposed model considers a novel selective pressure brought about, for example, by a recent change in the environment, which causes one or a few focal traits to be under directional selection but leaves selection on most traits unaltered. An allele experiences a selective benefit of $s_{\mathrm{f}}$ if it brings the focal trait(s) nearer its current optimum. This allele also has pleiotropic effects on several other traits, which are not directly measured. The sum of the pleiotropic selective effects on fitness is $s_{\mathrm{p}}$. Just as the average mutational effect on fitness is deleterious (Keightley \& Lynch 2003), I assume that the total of the pleiotropic effects of an allele has a negative impact on fitness $\left(s_{\mathrm{p}}<0\right.$; see also Hill \& Keightley 1988; Barton 1990; Keightley \& Hill 1990). Notice that I do not assume that the allele has a negative impact on all other traits; in fact, it may improve a subset of the non-focal traits, as long as the total pleiotropic effect is negative. The assumption that $s_{\mathrm{p}}<0$ seems reasonable if the novel selective pressure acts on only one (or a few) character traits and if the population is otherwise well adapted to its circumstances such that most rare alleles that affect fitness are deleterious. 
This model is similar in spirit to previous quantitative genetics models that decompose selection onto more than one trait axis. In models by Lande (1980), Turelli (1985), Wagner (1989), Slatkin \& Frank (1990) and others, alleles at a locus are assumed to affect multiple traits, each of which is under stabilizing selection. These studies have assessed the effects of pleiotropy on the maintenance of genetic variability for a focal trait at mutation-selection balance. Interestingly, whether or not mutation-selection balance is able to account for the observation that quantitative traits often exhibit high heritability despite the appearance of strong stabilizing selection is sensitive to the underlying genetic assumptions of the model (Zhang \& Hill 2002 and references therein). Much higher variance in a focal trait can be maintained at equilibrium when a pleiotropic trait experiences balancing selection (Robertson 1956; Bulmer 1973; Gillespie 1984; Barton 1990), although it is difficult to reconcile observed heritabilities with the segregation load that would be induced (Barton 1990).

As in the current paper, other quantitative genetic studies have summarized the pleiotropic effects of an allele by their net deleterious effect on fitness (Hill \& Keightley 1988; Barton 1990; Keightley \& Hill 1990; Kondrashov \& Turelli 1992). These studies have shown that pleiotropy tends to induce stabilizing selection on a focal trait, because individuals with multiple deleterious mutations also tend to have more extreme values of the focal trait. As a consequence, the strength of pleiotropic selection and the correlation between the effects of mutations on the pleiotropic and focal trait axes shape the amount of variation maintained in the focal trait at equilibrium. Furthermore, Hill \& Keightley (1988) examined the influence of deleterious pleiotropic effects on the response to directional selection in a quantitative genetics model, assuming that selection coefficients for the pleiotropic effect and the direct effect were drawn from exponential distributions, finding that the response to directional selection is lowered by pleiotropy, substantially so when the mean pleiotropic effect on fitness is large relative to the mean direct selective effect (see their table 1). A similar result was observed by Baatz \& Wagner (1997) in a 'corridor' model, in which stabilizing selection acts along one trait axis and directional selection along another (see their table 5). These studies also show that strong directional selection, as is typically encountered in artificial selection experiments, will tend to overwhelm the effects of pleiotropy, causing selection to proceed unencumbered (Hill \& Keightley 1988; Barton 1990; Baatz \& Wagner 1997).

In contrast to the above papers, the current paper focuses on the selective advantage and fate of an allele that has a favourable effect on a focal trait in the presence of deleterious pleiotropy, in an attempt to improve our understanding of the features that should be expected of alleles that spread in response to selection. Among alleles having a positive fitness effect through the focal trait $\left(s_{\mathrm{f}}>0\right)$, the distribution of pleiotropic effects remains unknown, so I will seek results that apply over a broad array of possible distributions. I will examine the effects of pleiotropy on alleles with known direct selective benefit $\left(s_{\mathrm{f}}\right)$, but I will describe generalizations to an unknown distribution of direct effects in $\ 2$. The crux of the analysis is the recognition that the total selection coefficient acting on the allele, $s_{\mathrm{t}}=s_{\mathrm{f}}+s_{\mathrm{p}}$, must remain positive for the allele to spread within a large population. As will be shown, this conditional statement $\left(s_{\mathrm{t}}>0\right)$ allows us to predict more about pleiotropic effects than one might initially think.

\section{(a) Fraction of alleles that are beneficial overall}

First consider the fraction, $F$, of alleles whose total selection coefficient is positive $\left(s_{t}>0\right)$. Here, we focus on alleles that are in sufficient copy number that they will spread to fixation if they are beneficial overall; we refer to such alleles as 'segregating alleles' in contrast to new mutations, which will be treated in later sections that incorporate fixation probabilities. Let $f\left(s_{\mathrm{p}}\right)$ equal the probability density function of pleiotropic effects for segregating alleles, where the net effect of pleiotropy is assumed to be detrimental (i.e. $f\left(s_{\mathrm{p}}\right)=0$ for $s_{\mathrm{p}}>0$ ), and the mean pleiotropic effect is defined as $-\mu$.

A fundamental problem is that we have few data about the form of the distribution of pleiotropic effects. Nevertheless, surprisingly general results can be derived for nearly any distribution when pleiotropy is strong, such that the pleiotropic effect on fitness often overwhelms direct selection on the focal trait. For an allele to have a beneficial effect overall $\left(s_{\mathrm{t}}>0\right)$, the negative pleiotropic effects of the allele on fitness, $s_{\mathrm{p}}$, must be less than the direct beneficial effect on the focal trait (i.e. $s_{\mathrm{p}}$ must fall between $-s_{\mathrm{f}}$ and 0 ). Thus, the fraction of alleles that remain beneficial overall is

$$
\begin{aligned}
& F=\int_{-s_{\mathrm{f}}}^{0} f\left(s_{\mathrm{p}}\right) \mathrm{d} s_{\mathrm{p}}, \\
& =s_{\mathrm{f}} f(0)-\frac{1}{2} s_{\mathrm{f}}^{2} f^{(i)}(0)+O\left(s_{\mathrm{f}}^{3}\right),
\end{aligned}
$$

where $f^{(i)}(0)$ represents the $i$ th derivative of $f\left(s_{\mathrm{p}}\right)$ with respect to $s_{\mathrm{p}}$ evaluated at $s_{\mathrm{p}}=0$. (Technically, because $f\left(s_{\mathrm{p}}\right)$ does not exist for positive $s_{\mathrm{p}}$ values, equation (1.2) is obtained by replacing the limits of integration of equation (1.1) with $-s_{\mathrm{f}}$ to $-\varepsilon$, performing a Taylor series of equation (1.1) with respect to $-s_{\mathrm{f}}$, and taking the limit as $-\varepsilon$ goes to 0 . As with all of the following general results, the higher-order terms in the Taylor series may be ignored only if the derivatives of the probability density function describing pleiotropic effects are well behaved near 0 . Specifically, $s_{\mathrm{f}}^{i+1} f^{(i)}(0) / i$ ! must be negligible relative to the kept terms, which is true when directional selection $\left(s_{\mathrm{f}}\right)$ is weak relative to pleiotropic selection (so that the distribution of pleiotropic effects does not change dramatically in height between $-s_{\mathrm{f}}$ and the origin). As will be discussed later in $₫ 1 \mathrm{~b}$, the Taylor series approximation fails for distributions such as the gamma distribution, whose height changes dramatically near 0 .

\section{(i) Implication 1}

When directional selection is weak relative to pleiotropic selection, the fraction of alleles that is beneficial overall is $s_{\mathrm{f}} f(0)$ to leading order in $s_{\mathrm{f}}$. This fraction does not depend on the mean pleiotropic effect but rather on the shape of the pleiotropic distribution near $0, f(0)$, which is much harder to estimate. Furthermore, the fraction of alleles that is beneficial overall depends linearly on the strength of selection on the focal trait when direct selection is weak. 
Table 1. The average pleiotropic effect on fitness of an allele with direct effect, $s_{\mathrm{f}}$, given that the total effect on fitness of the allele is beneficial $\left(\bar{s}_{\mathrm{p} \mid \text { beneficial }}\right)$.

(The pleiotropic effect, $s_{\mathrm{p}}$, is assumed to be negative and drawn from the given distribution with mean $-\mu$. The results for strong pleiotropy assume that $s_{\mathrm{f}} \ll|\mu| . M$ and $S$ are the mean and standard deviation of a normal distribution, which is then truncated above $0 . C$ is the coefficient of variation, $\Gamma[a]$ is the Euler gamma function, $\Gamma[a, x]$ is the incomplete gamma function, and Erf is the error function (see Abramowitz \& Stegun (1972) for definitions and simplifications involving these functions).)

\begin{tabular}{ll}
\hline distribution & general case $\bar{s}_{\mathrm{p} \mid \text { beneficial }}$ \\
\hline
\end{tabular}

uniform $\quad \begin{cases}-\frac{s_{\mathrm{f}}}{2} & \text { if } 0<s_{\mathrm{f}} \leqslant 2 \mu \ll 1 \\ -\mu & \text { if } 2 \mu \leqslant s_{\mathrm{f}} \ll 1\end{cases}$

$-\frac{s_{\mathrm{f}}}{2}$

exponential

$$
-\mu+\frac{s_{\mathrm{f}}}{\exp \left(s_{\mathrm{f}} / \mu\right)-1}
$$$$
-\frac{s_{\mathrm{f}}}{2}+O\left(s_{\mathrm{f}}^{2}\right)
$$

truncated normal

$$
\begin{aligned}
& M+\sqrt{\frac{2}{\pi}} S \frac{\exp \left[-M^{2} /\left(2 S^{2}\right)\right]-\exp \left[-\left(s_{\mathrm{f}}+M\right)^{2} /\left(2 S^{2}\right)\right]}{\operatorname{Erf}\left[\frac{M}{\sqrt{2} S}\right]-\operatorname{Erf}\left[\frac{s_{\mathrm{f}}+M}{\sqrt{2} S}\right]} \quad-\frac{s_{\mathrm{f}}}{2}+O\left(s_{\mathrm{f}}^{2}\right) \\
& -\mu+\frac{C^{2} \mu\left(\frac{s_{\mathrm{f}} \exp \left(-s_{\mathrm{f}} / \mu\right)}{C^{2} \mu}\right)^{1 / C^{2}}}{\Gamma\left[\frac{1}{C^{2}}\right]-\Gamma\left[\frac{1}{C^{2}}, \frac{s_{\mathrm{f}}}{C^{2} \mu}\right]} \quad-\frac{s_{\mathrm{f}}}{1+C^{2}}+O\left(s_{\mathrm{f}}^{2}\right)
\end{aligned}
$$

gamma

\section{(b) Average degree of pleiotropy among beneficial alleles}

Among alleles that are favourable overall, the countervailing selection pressure induced by pleiotropy is, on average

$\bar{s}_{\mathrm{p} \mid \text { beneficial }}=\int_{-_{\mathrm{f}}}^{0} s_{\mathrm{p}} f\left(s_{\mathrm{p}}\right) \mathrm{d} s_{\mathrm{p}} / F$.

Performing a Taylor Series of equation (1.3) under the assumption that selection on the focal trait is weak yields

$\bar{s}_{\mathrm{p} \mid \text { beneficial }}=-\frac{s_{\mathrm{f}}}{2}+\frac{s_{\mathrm{f}}^{2}}{12} \frac{f^{(1)}(0)}{f(0)}+O\left(s_{\mathrm{f}}^{3}\right)$.

Thus, the mean size of pleiotropic effects among alleles that are positively selected is, to leading order, $-s_{\mathrm{f}} / 2$, which does not depend on the unknown mean of the pleiotropic distribution, $-\mu$, or its shape!

\section{(i) Implication 2}

An allele that is favoured for its effects on a focal trait and that remains favoured despite negative pleiotropic effects will experience, on average, a total selection coefficient that is halved by pleiotropy, assuming that directional selection on the focal trait is weak relative to the average fitness effect of pleiotropy.

At the other extreme, if selection acting on the focal trait is strong relative to pleiotropy $\left(s_{\mathrm{f}} \gg|\mu|\right)$, then the average pleiotropic effect among favoured alleles approaches $-\mu$, as we would expect. Thus, under strong directional selection, the unknown mean of the distribution of pleiotropic effects becomes key to assessing the strength of the force counteracting selection on the focal trait.

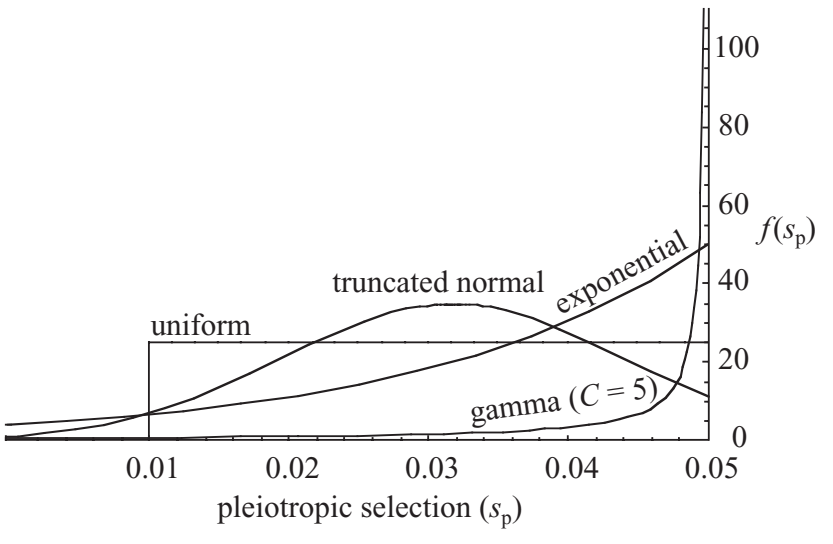

Figure 1. Probability density functions for the distributions of pleiotropic effects discussed in $₫ 1 \mathrm{~b}$. The total pleiotropic effect $\left(s_{\mathrm{p}}\right)$ is assumed to be negative with a mean of -0.02 .

The normal distribution is truncated 1.5 standard deviations above the mean at 0 .

To understand these results more fully, we now consider several possible distributions describing the magnitude of pleiotropic effects - uniform, exponential, gamma and truncated normal (figure 1). For each distribution, equation (1.3) is calculated exactly from the probability density function, $f\left(s_{\mathrm{p}}\right)$, yielding the average pleiotropic effect among beneficial alleles (table 1). Assuming that selection on the focal trait is weak relative to the extent of pleiotropy $\left(s_{\mathrm{f}} \ll|\mu|\right)$, the mean magnitude of pleiotropic effects among alleles that are positively selected is to leading order $-s_{\mathrm{f}} / 2$ for every one of these distributions except the gamma distribution. The reason that this simple result is obtained for distributions that differ so greatly in shape is that only a portion of the distribution matters: between 
(a)
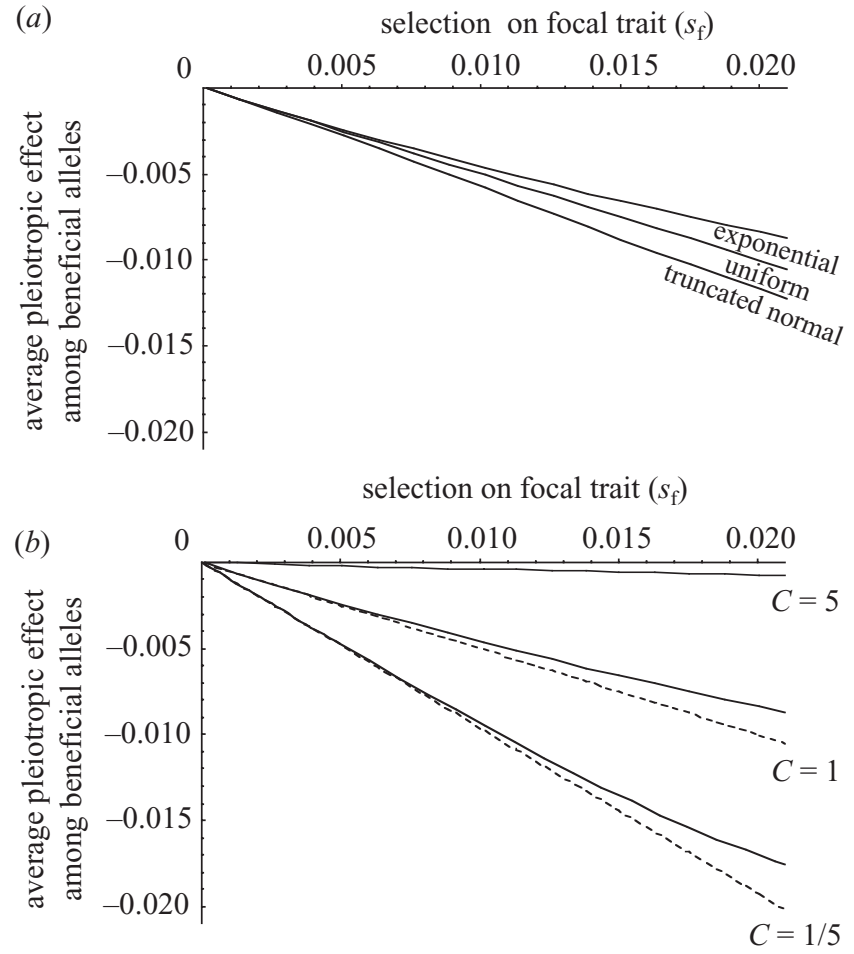

Figure 2. The average pleiotropic effect among alleles whose total selection coefficient remains positive $\left(\bar{s}_{\mathrm{p} \mid \text { beneficial }}\right)$ as a function of the strength of direct selection acting on the focal trait $\left(s_{\mathrm{f}}\right)$. In each case, the mean of the pleiotropic distribution is set to $-\mu=-0.02$. (a) $\bar{s}_{\mathrm{p} \text { |beneficial }}$ for the exponential, uniform and truncated-normal distributions illustrated in figure 1 . Note that $\bar{s}_{\mathrm{p} \mid \text { beneficial }}$ is well approximated by $-s_{\mathrm{f}} / 2$ (the line for the uniform distribution) even when direct selection is nearly equal to the average strength of pleiotropic selection $s_{\mathrm{f}} \approx \mu$. (b) $\bar{s}_{\mathrm{p} \mid \text { beneficial }}$ for the gamma distribution with coefficient of variation set to 5 ( $\mathrm{L}$ shaped), 1 (exponential) and 1/5 (bell shaped). The dashed lines show the approximation $\bar{s}_{\mathrm{p} \mid \text { beneficial }} \approx s_{\mathrm{f}}\left(1+C^{2}\right)$ (the dashed and solid curved lines are indistinguishable for $C=5)$.

$-s_{\mathrm{f}}$ and 0 . Even if the distribution is highly non-uniform outside of this range, as long as the probability density changes little within this region, then the results obtained for a uniform distribution $\left(\bar{s}_{\mathrm{p} \mid \text { beneficial }}=-s_{\mathrm{f}} / 2\right)$ provide an adequate estimate of the mean pleiotropic effect for favoured alleles, as shown in figure $2 a$.

The only distribution that yields a different result is the gamma distribution. The probability density function for a gamma changes dramatically near 0 unless the coefficient of variation, $C$ (the standard deviation over the mean of the distribution of pleiotropic effects), is 1. However, the approximations in equations (1.2) and (1.4) hold only when the distribution function has finite derivatives and a finite positive value at 0 , which is not the case for the gamma distribution. Instead, for the gamma distribution, the fraction of alleles that is beneficial is $F=1-$ $\Gamma\left[1 / C^{2}, s_{\mathrm{f}} /\left(C^{2} \mu\right)\right] / \Gamma\left[1 / C^{2}\right]$. When selection on the focal trait is weak relative to pleiotropy $\left(s_{\mathrm{f}} \ll \min \left[C^{2} \mu, 1\right]\right)$, this fraction is approximately $\left(s_{\mathrm{f}} /\left(C^{2} \mu\right)\right)^{1 / C^{2}} / \Gamma\left[1 /\left(1+C^{2}\right)\right]$, which rises with the coefficient of variation, such that virtually no alleles are beneficial overall when $C \approx 1 / 2$, whereas almost all alleles are beneficial when $C \approx 20$, reflecting the greater abundance of mutations with negligible pleiotropic effect.

For the gamma distribution, the mean magnitude of the fitness effects of pleiotropy among positively selected alleles is $\bar{s}_{\mathrm{p} / \text { beneficial }}=-s_{\mathrm{f}} /\left(1+C^{2}\right)$ to leading order in $s_{\mathrm{f}}$ when direct selection is weak relative to the average effects of pleiotropy. When $C$ is near 1 , the gamma distribution is approximately exponential, and $\bar{s}_{\mathrm{p} / \text { beneficial }}$ is again $\approx-s_{\mathrm{f}} / 2$. If, however, $C$ is much greater than 1 , the distribution of pleiotropic effects is very $L$ shaped and falls rapidly near 0 , so that $-s_{\mathrm{f}} / 2$ overestimates the magnitude of pleiotropic effects among favourable alleles. Conversely, if $C$ is much smaller than 1 , the distribution of pleiotropic effects rises rapidly near 0 , and $-s_{\mathrm{f}} / 2$ underestimates the magnitude of pleiotropic effects (see figure $2 b$ ).

Data based on mutation-accumulation experiments in Drosophila melanogaster suggest that the coefficient of variation for new random mutations varies from 2 to 5 for bristle traits and viability (Keightley 1994). These estimates are, however, based on the full spectrum of mutations. We are only interested in those alleles that have some positive effect on the focal trait $\left(s_{\mathrm{f}}>0\right)$; such alleles may be more likely to affect a broader array of pleiotropic characters than are random mutations, many of which may be truly silent. Therefore the coefficient of variation for the distribution of pleiotropic effects among alleles that have some effect on a given trait is probably overestimated by mutation-accumulation experiments. This suggests that $C$ values may be near enough to 1 for $\bar{s}_{\mathrm{p} \mid \text { beneficial }} \approx$ $-s_{\mathrm{f}} / 2$ to be realistic even for a gamma distribution of pleiotropic effects.

\section{(c) Average fixation probability}

Mutations that are beneficial to a trait under novel selective pressures may fail to become established within a population for two reasons. First, negative pleiotropy can so drastically reduce the fitness of the mutation that it becomes deleterious overall $\left(s_{\mathrm{t}}<0\right)$. Second, even those new alleles that are beneficial overall may be lost from a population when rare owing to the erratic and uncertain nature of survival and reproduction. Haldane (1927) demonstrated that if the number of offspring per parent is Poisson distributed, the probability that a single mutant allele within a population will leave descendants over an indefinitely long period of time is approximately $2 s_{\mathrm{t}}$, which is accurate when $1 / N_{\mathrm{e}} \lesssim s_{\mathrm{t}} \lesssim 0.1$, where $N_{\mathrm{e}}$ is the effective population size. Let us now calculate the effects of pleiotropy on the fixation probability of a mutation that arises in a single copy and that is beneficial for the focal trait, assuming that the population is large enough that alleles whose total effects are deleterious are unable to fix. Note that the distribution of pleiotropic effects among mutations that appear in a single copy $\left(f_{\text {new }}\left(s_{\mathrm{p}}\right)\right.$ with mean $\left.-\mu_{\text {new }}\right)$ need not equal the distribution of pleiotropic effects among all segregating alleles $\left(f\left(s_{\mathrm{p}}\right)\right)$ discussed in $\int 1$ b,c. In fact, we would expect new mutations to have more severe pleiotropic effects, on average, than alleles segregating at higher copy numbers, which have survived the test of past selection (i.e. we expect $-\mu_{\text {new }}<-\mu$ ).

Among single-copy mutant alleles with direct effect $s_{\mathrm{f}}$, the average probability of fixation is given by: 
Table 2 . The average probability of fixation of single-copy alleles with direct effect $s_{\mathrm{f}}$

$\left(s_{\mathrm{p}}\right.$ is assumed to be negative and drawn from the given distribution $\left(f_{\text {new }}\right)$ with mean $-\mu_{\text {new }}$. To simplify the table, we drop the subscript 'new' from $f$ and $\mu$. Results followed by ${ }^{\ddagger}$ are exact solutions to equation (1.5).)

\begin{tabular}{|c|c|c|}
\hline distribution & average probability of fixation & \\
\hline uniform & $\left\{\begin{array}{l}\frac{1}{2 \mu} s_{\mathrm{f}}^{2}=f(0) s_{\mathrm{f}}^{2 \ddagger} \\
2\left(s_{\mathrm{f}}-\mu\right)^{\ddagger}\end{array}\right.$ & $\begin{array}{l}\text { if } 0<s_{\mathrm{f}} \leqslant 2 \mu \ll 1 \\
\text { if } 2 \mu \leqslant s_{\mathrm{f}} \ll 1\end{array}$ \\
\hline exponential & $\left\{\begin{array}{l}\frac{1}{\mu} s_{\mathrm{f}}^{2}=f(0) s_{\mathrm{f}}^{2} \\
2\left\{s_{\mathrm{f}}-\left[1-\exp \left(-\frac{s_{\mathrm{f}}}{\mu}\right)\right] \mu\right\}^{\ddagger} \\
2\left(s_{\mathrm{f}}-\mu\right)\end{array}\right.$ & $\begin{array}{l}\text { if } 0<s_{\mathrm{f}} \ll \mu \ll 1 \\
\text { if } 0<s_{\mathrm{f}} \ll 1 \\
\text { if } \mu \ll s_{\mathrm{f}} \ll 1\end{array}$ \\
\hline truncated normal & $\left\{\begin{array}{l}\sqrt{\frac{2}{\pi}} \frac{\exp \left[-M^{2} /\left(2 s^{2}\right)\right]}{S\left(1-\operatorname{Erf}\left[\frac{M}{\sqrt{2} S}\right]\right)^{2}} s_{\mathrm{f}}^{2}=f(0) s_{\mathrm{f}}^{2} \\
2 S \sqrt{\frac{2}{\pi}} \frac{\exp \left[-M^{2} /\left(2 S^{2}\right)\right]-\exp \left[-\left(s_{\mathrm{f}}+M\right)^{2} /\left(2 S^{2}\right)\right]}{\left(1-\operatorname{Erf}\left[\frac{M}{\sqrt{2} S}\right]\right)}\left(\frac{s_{\mathrm{f}}+\bar{s}_{\mathrm{p} \mid \text { beneficial }}}{M-\bar{s}_{\mathrm{p} \mid \text { beneficial }}}\right) \\
2\left(s_{\mathrm{f}}-\mu\right)\end{array}\right.$ & $\begin{array}{l}\text { if } 0<s_{\mathrm{f}} \ll 1 \\
\text { if } \mu \ll s_{\mathrm{f}} \ll 1\end{array}$ \\
\hline gamma & $\left\{\begin{array}{l}\frac{2 s_{\mathrm{f}}}{\Gamma\left[2+\frac{1}{C^{2}}\right]}\left(\frac{s_{\mathrm{f}}}{C^{2} \mu}\right)^{1 / C^{2}} \neq f(0) s_{\mathrm{f}}^{2} \\
\frac{2 \mu}{\Gamma\left[1+\frac{1}{C^{2}}\right]}\left(\frac{s_{\mathrm{f}}+\bar{s}_{\mathrm{p} \mid \text { beneficial }}}{\mu+\bar{s}_{\mathrm{p} \mid \text { beneficial }}}\right)\left(\frac{s_{\mathrm{f}} \exp \left(-s_{\mathrm{f}} / \mu\right)}{C^{2} \mu}\right)^{1 / C^{2}} \\
2\left(s_{\mathrm{f}}-\mu\right)\end{array}\right.$ & $\begin{array}{l}\text { if } 0<s_{\mathrm{f}} \ll 1 \\
\text { if } C^{2} \mu \ll s_{\mathrm{f}} \ll 1\end{array}$ \\
\hline
\end{tabular}

$\overline{P_{\text {fix }}}=\int_{s_{\mathrm{f}}}^{0} 2\left(s_{\mathrm{p}}+s_{\mathrm{f}}\right) f_{\text {new }}\left(s_{\mathrm{p}}\right) \mathrm{d} s_{\mathrm{p}}$.

Performing a Taylor series of equation (1.5) under the assumption that selection on the focal trait is weak yields

$\overline{P_{\text {fix }}}=f_{\text {new }}(0) s_{\mathrm{f}}^{2}-\frac{1}{3} f_{\text {new }}^{(1)}(0) s_{\mathrm{f}}^{3}+O\left(s_{\mathrm{f}}^{4}\right)$.

\section{(i) Implication 3}

When pleiotropy is strong, the probability that a mutation that improves a focal trait will become established within a population is extremely small, being proportional to the square of its direct selective effect, $s_{\mathrm{f}}^{2}$. Thus, most potentially beneficial alleles will not become established because of the negative effects of pleiotropy as well as the vagaries of random genetic drift.

Exact values of $\overline{P_{\text {fix }}}$ are presented in table 2 for the uniform, exponential, gamma and truncated-normal distributions. If pleiotropic effects are large, the ability to fix alleles beneficial to the focal trait is strongly reduced. For example, if selection on a focal trait is, on average, $10 \%$ of the magnitude of pleiotropic selection $\left(s_{\mathrm{f}}=0.1 \mu_{\text {new }}\right)$ the fixation probability of an allele that improves the focal trait is less than $5 \%$ of its expected value $\left(2 s_{\mathrm{f}}\right)$ in the absence of pleiotropy for every distribution except for L-shaped gamma distributions (with $C \geqslant 1.2$ ). Conversely, if pleiotropic effects are weak then the average probability of fixation is $2\left(s_{\mathrm{f}}-\mu_{\text {new }}\right)$, i.e. reduced by the average negative effect of pleiotropy, as expected.

\section{(d) Average degree of pleiotropy among fixed mutations}

Among those few alleles that do succeed in fixing, the average effect of pleiotropy can be calculated by weighting the distribution of pleiotropic effects by their fixation probability:

$\bar{s}_{\mathrm{p} \mid \text { fixation }}=\int_{-_{\mathrm{f}}}^{0} 2\left(s_{\mathrm{p}}+s_{\mathrm{f}}\right) s_{\mathrm{p}} f_{\text {new }}\left(s_{\mathrm{p}}\right) \mathrm{d} s_{\mathrm{p}} \overline{P_{\text {fix }}}$.

Performing a Taylor series of equation (1.7) under the assumption that selection on the focal trait is weak yields

$\bar{s}_{\mathrm{p} \mid \text { fixation }}=-\frac{s_{\mathrm{f}}}{3}+\frac{s_{\mathrm{f}}^{2}}{18} \frac{f_{\text {new }}^{(1)}(0)}{f_{\text {new }}(0)}+O\left(s_{\mathrm{f}}^{3}\right)$.

Again, the average effect of pleiotropy among fixed mutations can be calculated exactly for the distributions shown in figure 1 using equation (1.7), which confirms that equation (1.8) provides a good approximation with 
extensive pleiotropy except for the case of a gamma distribution, in which case $-s_{\mathrm{f}} /\left(1+2 C^{2}\right)$ provides a better approximation.

\section{(i) Implication 4}

Among fixed mutations, pleiotropy reduces the total selection coefficient by one-third, on average, when the mutations improve a focal trait by an amount that is small relative to the average fitness effects of pleiotropy $\left(s_{\mathrm{f}} \ll \mu_{\text {new }}\right)$. The average effect of pleiotropy among alleles that succeed in fixing $\left(-s_{\mathrm{f}} / 3\right)$ is less than among alleles that are beneficial overall $\left(-s_{\mathrm{f}} / 2\right)$ because alleles with weaker deleterious pleiotropic effects are more likely to fix.

\section{(e) Empirical implications}

Data are accumulating from quantitative trait locus (QTL) experiments that estimate the number and effect size of loci contributing to a quantitative trait difference between two populations. Typically, these experiments chose one or a few focal traits and estimate the distribution of alleles contributing to the parental difference in these traits. The model of Fisher (1930) has been used to predict the shape of this distribution, yielding a surprisingly robust result: the shape is typically exponential (Orr 1998, 1999; Griswold \& Whitlock 2003). The results of this paper can also be used to generate an expectation against which QTL data may be compared. Consider a QTL study that estimates the loci contributing to differences between two populations in a focal trait that has been subject to directional selection in the past. If this directional selection has been weak relative to the pleiotropic fitness effects of alleles at potential QTL, we can use table 2 to determine how pleiotropy shapes the distribution of alleles that can fix within a population. To be concrete, let us assume that, among all possible mutations that improve the focal trait, the distribution of direct fitness effects, $g\left(s_{\mathrm{f}}\right)$, is gamma distributed with mean $\mu_{\mathrm{f}}$ and coefficient of variance $C_{\mathrm{f}}$, whereas the distribution of deleterious pleiotropic fitness effects is uncorrelated and gamma distributed with mean $-\mu_{\mathrm{p}}$ and coefficient of variance $C_{\mathrm{p}}$. Further assume that the distributions of direct and pleiotropic effects have been relatively constant over the course of selection, which is reasonable if each mutational step is small relative to the distance from a fixed optimum (as shown in the simulation study of Griswold \& Whitlock (2003)) or if the optimum is moving away along the focal trait axis.

When selection on the focal trait is weak $(0<$ $\left.s_{\mathrm{f}} \ll \min \left[C_{\mathrm{p}}^{2} \mu_{\mathrm{p}}, 1\right]\right)$, the fixation probability of single-copy mutations is proportional to $s_{\mathrm{f}}^{1+1 / C_{\mathrm{p}}^{2}}$ (table 2). Using this fixation probability to weight the distribution of direct fitness effects $g\left(s_{\mathrm{f}}\right)$, we find that the distribution of effects on the focal trait among alleles that can successfully fix within a population is gamma distributed with mean

$\mu_{\mathrm{f}}\left(1+C_{\mathrm{f}}^{2}+\frac{C_{\mathrm{f}}^{2}}{C_{\mathrm{p}}^{2}}\right)$.

The mean is higher than the mean effect of new mutants that improve the trait $\left(\mu_{\mathrm{f}}\right)$ because alleles with a large beneficial effect are more likely to survive loss while rare (contributing the $C_{\mathrm{f}}^{2}$ term, as found by Otto \& Jones (2000), assuming that pleiotropic effects were negligible) and because alleles with a large beneficial effect are more likely to be beneficial overall (contributing the $C_{\mathrm{f}}^{2} / C_{\mathrm{p}}^{2}$ term, which remains even if fixation of beneficial alleles is assured). The coefficient of variation for the effects of fixed alleles on the focal trait becomes

$$
\sqrt{\frac{C_{\mathrm{f}}^{2}}{1+C_{\mathrm{f}}^{2}+C_{\mathrm{f}}^{2} / C_{\mathrm{p}}^{2}}},
$$

which is always smaller than 1 and smaller than $C_{\mathrm{f}}$. (If the distribution of pleiotropic effects is not gamma but has a finite slope and intercept, then the fixation probability is proportional to $s_{\mathrm{f}}^{2}$ rather than $s_{\mathrm{f}}^{1+1 / C_{\mathrm{p}}^{2}}$ (table 2), and $C_{\mathrm{p}}$ may be set to 1 in equations (1.9) and (1.10).) This generalizes the result of Kimura (1983) that the distribution of fixed alleles is expected to be bell shaped with coefficient of variation $\sqrt{1 / 2}$ if new mutations have exponentially distributed fitness effects $\left(C_{\mathrm{f}}=1\right)$ to describe gamma distributions that are not exponential in shape and to include deleterious pleiotropy.

As noted by Otto \& Jones (2000), the coefficient of variation among fixed mutations is $\sqrt{C_{\mathrm{f}}^{2} /\left(1+C_{\mathrm{f}}^{2}\right)}$ when pleiotropy is negligible. Interestingly, this implies that the distribution of fixed effects will be nearly exponential (coefficient of variation near 1 ) if the direct effects of new mutations on the focal trait follow an L-shaped distribution $\left(C_{\mathrm{f}} \gg 1\right)$. Equation (1.10) indicates that this result continues to hold and pleiotropy will have little effect on the shape of the distribution of fixed alleles as long as the distribution of direct and pleiotropic effects are L-shaped $\left(C_{\mathrm{f}}, C_{\mathrm{p}} \gg 1\right)$, in which case most alleles with a positive total fitness effect have negligible pleiotropic effects sampled from the mode of the L-shaped distribution. If, however, pleiotropic effects follow a more bell-shaped distribution than direct effects $\left(C_{\mathrm{p}} \ll C_{\mathrm{f}}, 1\right)$, the coefficient of variation among fixed alleles also becomes bell shaped with coefficient of variation $\approx C_{\mathrm{p}}$, that is, pleiotropy strongly constrains the distribution of alleles that can spread and fix within a population.

Figure 3 contrasts these two cases, illustrating the effects of pleiotropy when $C_{\mathrm{p}}=2$ and 0.5 . It is interesting to note that, although the underlying model and key assumptions are substantially different from the analyses of adaptation using the model of Fisher (1930; Orr 1998, 1999), we again predict a nearly exponential distribution for the effect of fixed alleles on a focal trait as long as the distributions of direct and pleiotropic effects of new mutations are fairly leptokurtic (equation (1.10) is near 1 for $C_{\mathrm{f}}, C_{\mathrm{p}} \gg 1$ ). If, however, the distribution of either the direct effects or the pleiotropic effects is more bell shaped $\left(C_{\mathrm{f}}\right.$ or $\left.C_{\mathrm{p}}<1\right)$ or changes little in height near 0 (as with the exponential, uniform and truncated-normal distributions), we would expect a bell-shaped distribution (with a coefficient of variation that is $\leqslant \sqrt{1 / 2}$ ) among the effects on a focal trait of alleles that have fixed within a lineage. The key assumptions made here are that pleiotropy is strong relative to the direct effects of selection and that the total pleiotropic effect is always deleterious.

The results of this paper also have implications for what we might expect to observe in artificial selection experiments, as long as two requirements are met as follows:

(i) most of the response to selection must be owing to the spread of alleles that become beneficial under artificial selection and not to shifts in the frequency 


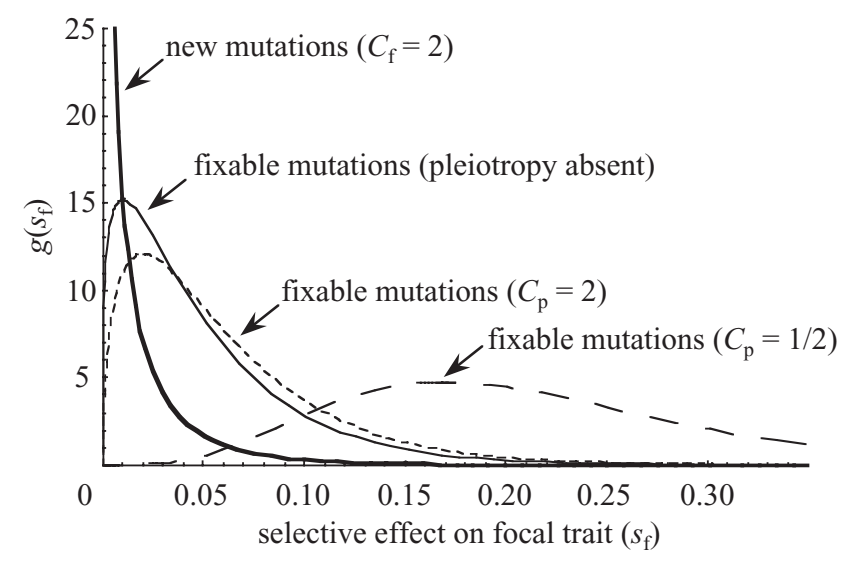

Figure 3. The impact of strong deleterious pleiotropy on the distribution of allelic effects on a focal trait predicted by equations (1.9) and (1.10). The distribution among new single-copy mutations, $g\left(s_{\mathrm{f}}\right)$, is assumed to be gamma with mean $\mu_{\mathrm{f}}=0.01$ and $C_{\mathrm{f}}=2$ (thick solid curve). In the absence of pleiotropy, the distribution among fixed alleles becomes nearly exponential in shape with a coefficient of variation of $\sqrt{4 / 5}=0.89$ (thin solid curve). When pleiotropic effects are $\mathrm{L}$ shaped $\left(C_{\mathrm{p}}=2\right)$, the distribution among fixed alleles remains nearly exponential, with a coefficient of variation of $\sqrt{4 / 6}=0.82$ (short dashed curve). When pleiotropic effects are bell shaped $\left(C_{\mathrm{p}}=1 / 2\right)$, however, the distribution among fixed alleles becomes bell shaped, with a coefficient of variation of only $\sqrt{4 / 21}=0.44$ (long dashed curve).

of alleles that have remained deleterious but with an altered selection coefficient; and

(ii) artificial selection must be weak relative to the average strength of pleiotropy.

The first requirement is unlikely to be satisfied in large populations with substantial standing variation in fitness, because a large fraction of the genetic variance in fitness may be generated by deleterious alleles whose frequencies shift during the course of artificial selection. If the populations are initially bottlenecked, however, the spread of newly arising beneficial alleles should account for most of the response to selection. Assuming that the populations are initially bottlenecked and that artificial selection is weak relative to pleiotropy, the average pleiotropic effect on fitness will be $\bar{s}_{\mathrm{p} \mid \text { fixation }} \approx-s_{\mathrm{f}} / 3$ among new mutations that spread to fixation (equation (1.8)). Consequently, the total selection pressure in the presence of artificial selection will be $s_{\mathrm{t}}=s_{\mathrm{f}}+\bar{s}_{\mathrm{p} \text { fixation }} \approx 2 / 3 s_{\mathrm{f}}$. After artificial selection is relaxed, we would expect this to fall to $s_{\mathrm{t}}=\bar{s}_{\mathrm{p} \mid \text { fixation }} \approx-s_{\mathrm{f}} / 3$. In other words, if artificial selection is applied for several generations and then suspended, the population should return towards the original mean at half the rate of advance under artificial selection. This prediction refers to the slope of the trait response over time measured near the point at which selection is relaxed, so that the allele frequencies of the favourable alleles before and after the cessation of selection are similar. This prediction is difficult to test using the current literature on artificial selection experiments, however, because artificial selection cannot be assumed weak relative to pleiotropy.

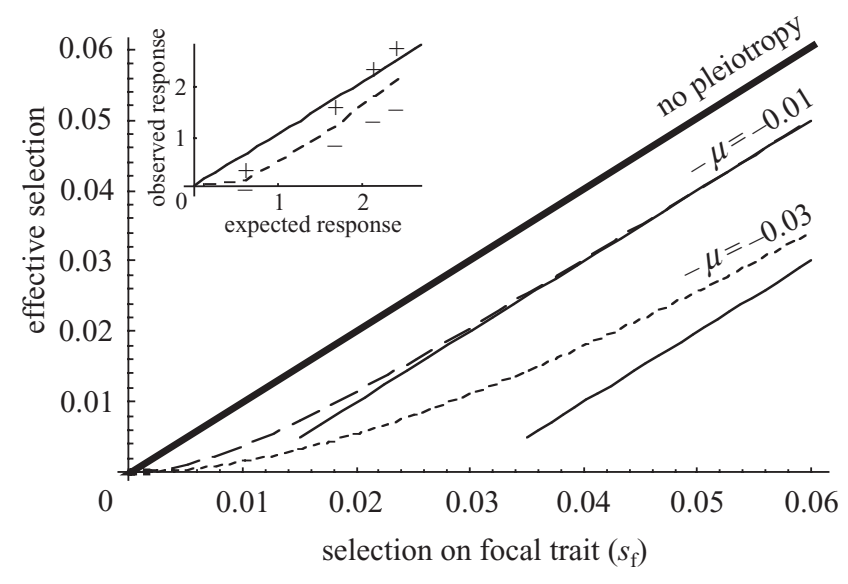

Figure 4. The effective strength of selection in the presence of pleiotropy as a function of the applied strength of selection in a short-term artificial selection experiment. The plot illustrates the effective selection coefficient, defined as the averaged value of the total selection coefficient when it is positive $\left(s_{\mathrm{T}}\right.$ using equation (1.3)) times the probability that $s_{\mathrm{T}}$ is positive (equation (1.1)), versus the strength of artificial selection on the focal trait $\left(s_{\mathrm{f}}\right)$. Here, the negative effects of pleiotropy are described by an exponential distribution with mean $-\mu=-0.01$ (long dashed curve) and $-\mu=-0.03$ (short dashed curve). In the absence of pleiotropy, a $1: 1$

relationship would be expected (thick line). With pleiotropy, the effective selection is reduced, especially when selection is weak (near the origin). With stronger direct selection, i.e. when direct selection reaches approximately twice the average magnitude of pleiotropy, the curves describing the effective selection straighten out and have a slope of 1 (thin lines) but are shifted down by $\mu$. Because pleiotropy reduces the total effective selection, one could measure pleiotropy from artificial selection experiments on initially variable populations by plotting the response to selection $(R)$ divided by the heritability of a trait $\left(h^{2}\right)$ versus the applied selection differential $(S)$, which is expected to produce a line in the absence of pleiotropy (Falconer \& Mackay 1996). The sum total magnitude of pleiotropy acting against the alleles subject to directional selection can then be measured by how far below the $R / h^{2}=S$ line the observed $R / h^{2}$ falls once the curves straighten out. This pattern is illustrated in the inset figure using the data of Clayton et al. (1956) on the magnitude of the response to selection on bristle number in Drosophila melanogaster for up-selected lines $(+)$, downselected lines $(-)$ and their average (dashed curve).

One can assess the relative magnitudes of pleiotropy and artificial selection by using data from a series of artificial selection experiments conducted across a range of selection pressures. When directional selection is very weak relative to the average effects of pleiotropy, the total selection coefficient of favourable alleles is substantially reduced, and fewer alleles will be favourable overall. As direct selection becomes strong relative to pleiotropy $\left(s_{\mathrm{f}} \gg \mu\right)$, however, the response to selection becomes more nearly proportional to the strength of artificial selection. Therefore, if one plots the realized selection differential (= response/heritability) as a function of the selection differential, one can detect the effect of pleiotropy and estimate its magnitude, as illustrated in figure 4. Similar predictions about the response to selection with stronger directional selection were obtained by Hill \& Keightley (1988; their table 1) and Baatz \& Wagner (1997; their 
table 5). In particular, pleiotropy causes the realized selection differential to be a convex function of the applied selection differential. Such a pattern was observed in a selection experiment in D. melanogaster conducted by Clayton et al. (1956). After five generations of truncation selection on abdominal bristle number, Clayton et al. (1956) noticed that in both up-selected and down-selected lines 'the ratio of observed to predicted responses is highest in the 20/100 [strongly selected] lines and declines continually to the 20/25 [weakly selected] lines.' Although the authors say that 'it is difficult to suggest a reason for this', the convex pattern of response is consistent with pleiotropy (see inset in figure 4). Of course, selection acting upon linked loci will also interfere with the response to selection (Hill \& Robertson 1966), but the negative effects of hitchhiking will be negligible under weak selection because recombination has more time to uncouple the loci when alleles change slowly in frequency (Barton 1995) and will become substantial only under high-selection differentials. Consequently, selective interference among loci would lead the response curve to rise linearly at first and then fall off, forming a concave function. Similarly, one might expect stabilizing natural selection to lead to a concave response function as populations under stronger selection will have evolved further from their original trait values. Thus, the shape of the response curve can inform us about the relative importance of pleiotropy versus selective interference among loci and/or stabilizing selection, all of which are factors that limit the response of populations to selection.

Although pleiotropy is less important relative to direct selection when direct selection is strong, it does not follow that pleiotropy is less important in an absolute sense. In fact, if a phenotypic character is selected until it changes from $P_{0}$ to $P_{1}$ by weak and by strong direct selection, one would expect more deleterious side effects in the case of stronger direct selection. This is because stronger direct selection allows the spread of alleles with more deleterious pleiotropic effects (i.e. the fraction, $F$, of the distribution of pleiotropic effects allowing a positive overall fitness effect is larger). Consequently, if one were to perform such an artificial selection experiment and relax selection, there should be a faster return to the original mean given the current heritability in the more strongly selected lines. Hitchhiking should generate a similar pattern, however, in that stronger selection will have dragged along deleterious alleles over a wider range of the genome and with more detrimental effects on fitness than weak selection. By contrast, direct natural selection on the trait (e.g. stabilizing selection) would not be sensitive to the history of selection and predicts no difference in response among replicates with the same phenotype $\left(P_{1}\right)$.

\section{DISCUSSION}

...it takes all the running you can do, to keep in the same place. If you want to get somewhere else, you must run at least twice as fast as that!

The Red Queen

(Through the looking glass, Carroll 1965, p. 152)

Pleiotropy is one of the most commonly observed attributes of genes. Yet the extent and influence of pleiotropy has been underexplored in population genetics models. In this paper, I have quantified how much pleiotropy can be expected to occur at the level of fitness among alleles that spread in response to directional selection. General insights into the effects of pleiotropy can be obtained by focusing on alleles that improve one or a few focal traits undergoing directional selection in an organism that is otherwise well adapted to its environment and by assuming that the sum of pleiotropic effects on fitness is negative and large relative to selection on the focal trait(s). Under this scenario, the negative fitness effects of pleiotropy, on average, halve the total selection coefficient among alleles whose overall fitness effect is beneficial $\left(\bar{s}_{\mathrm{p} \mid \text { beneficial}}\right.$, table 1$)$. This result is the outcome of the fact that we are only interested in those alleles whose total selection coefficient, including direct $\left(s_{\mathrm{f}}\right)$ and pleiotropic effects $\left(s_{\mathrm{p}}\right)$, remains positive $\left(s_{\mathrm{f}}+s_{\mathrm{p}}>0\right)$. This requirement bounds the distribution of potential pleiotropic effects to those lying between $-s_{\mathrm{f}}$ and 0 , and, for many distributions, $-s_{\mathrm{f}} / 2$ approximates the average value between these bounds (table 1, figure $2 a$ ). The net result is that for every two steps forward that direct selection takes, negative pleiotropic selection takes one step back. This result holds as long as the density of pleiotropic effects does not rise or fall dramatically near 0. If, however, there is an abundance of mutations with negligible pleiotropic effects (as for a gamma distribution with $C \gg 1$ ), then pleiotropy counteracts selection to a lesser degree, causing, on average, only $2 /\left(1+C^{2}\right)$ steps back for every two steps forward. The key restrictive assumption is that direct selection on the focal trait is weak relative to the distribution of deleterious pleiotropic effects. Such an assumption is less tenable for artificial selection than natural selection. It is also a more restrictive assumption for segregating alleles present in multiple copies than for new single-copy mutations, because alleles segregating at higher frequency will have passed through a selective sieve eliminating alleles with strong deleterious pleiotropic effects.

In this paper, I have focused on cases where the net effect of pleiotropy is always negative. It is straightforward to extend these results to distributions that allow beneficial pleiotropic effects $\left(s_{\mathrm{p}}>0\right)$ as long as one specifies the shape of this distribution, $f\left(s_{\mathrm{p}}\right)$. Results are available upon request for a reflected exponential distribution and a normal distribution. No general implications emerged from these analyses, however. Instead, the average amount of pleiotropy among beneficial and among fixed beneficial alleles is sensitive to the fraction of pleiotropic effects that are positive and to the exact shape of the distribution.

The results have been derived ignoring selection at linked loci, but, in practice, it may be difficult to tease apart pleiotropic effects on fitness from selection acting upon linked loci (Hill \& Robertson 1966; Barton 1995). For tight linkage, however, the distribution of pleiotropic effects can be expanded to describe both the side effects of the focal allele and closely linked alleles at other loci. Thus, the results described in this paper apply to both pleiotropy and tightly linked variation, as long as the sum continues to reduce the fitness of the focal allele. Tightly linked variation will tend to cause fewer alleles to be beneficial overall (linked deleterious alleles will reduce $f(0)$ in equation (1.2) for $F$ even further), and we would expect negative pleiotropic effects and negative associations with 
other loci to halve the advantageous effects of those alleles that do rise in frequency in response to selection.

Thus far, I have focused on alleles with a given selective effect on the focal trait(s), $s_{\mathrm{f}}$. When considering an entire genome, however, each allele that affects the focal trait will have its own selective effect. To get the average effect of pleiotropy across a genome, one must integrate the results obtained in this paper over the distribution of direct selection coefficients, $g\left(s_{\mathrm{f}}\right)$. The main approximations derived within this paper would continue to hold as long as, across this distribution, the strength of selection acting directly on the focal traits is weak relative to the average strength of pleiotropy. This requirement is not overly restrictive, however, because the magnitude of selection on the focal trait and pleiotropic traits may often be positively correlated, as has been observed for Pelement insertions (Mackay et al. 1992). It is reasonable to expect such a positive correlation, because mutations with a strong effect on the focal trait will more often alter amino acid sequences than be silent and may occur more often in regulatory regions than in coding regions; such mutations are also more likely to have stronger pleiotropic effects. As long as it is the case that, for the set of alleles whose selective effect on the focal trait is near $s_{\mathrm{f}}$, there is a distribution of pleiotropic effects whose mean is large relative to $s_{\mathrm{f}}$ and whose height changes by a small proportion between $f(0)$ and $f\left(-s_{\mathrm{f}}\right)$ (see $\int 1 \mathrm{~b}-\mathrm{d}$ for results that apply to a gamma distribution), then we can generalize the four main implications of this paper, which are as follows. The fraction of alleles that are beneficial overall will be approximately $\overline{s_{\mathrm{f}} f(0)}$ (that is, the average of $s_{\mathrm{f}} f(0)$ over the distribution $g\left(s_{\mathrm{f}}\right)$ ), which does not depend on the mean pleiotropic effect but rather on the shape of the pleiotropic distribution near 0 (implication 1 , see $\ 1 \mathrm{a}(\mathrm{i})$ ). Alleles that are favoured for their effects on a focal trait and that remain favoured despite negative pleiotropic effects will, on average, experience a total selection coefficient that is halved by pleiotropy (implication 2 , see $\int 1 \mathrm{~b}(\mathrm{i})$ ). The probability that mutations that improve a focal trait will become established within a population is extremely small, being proportional to the mean of their direct selective effect squared, $\overline{s_{\mathrm{f}}^{2}}$. Thus, most potentially beneficial alleles will not become established because of the negative effects of pleiotropy as well as the vagaries of random genetic drift (implication 3, see $₫ 1 \mathrm{c}(\mathrm{i})$ ). Among fixed mutations, pleiotropy will, on average, reduce the total selection coefficient by one-third (implication 4 , see $\ 1 \mathrm{~d}(\mathrm{i})$ ).

The author is grateful to A. Orr and M. Whitlock for conversations that inspired this work, to R. Gomulkiewicz, C. Griswold, T. Johnson, A. Orr, D. Schluter, M. Whitlock, G. Wagner and an anonymous referee for many helpful comments on the manuscript, and to D. Schluter for suggesting the title. This work was funded by the Natural Sciences and Engineering Research Council, Canada.

\section{REFERENCES}

Abramowitz, M. \& Stegun, I. A. 1972 Handbook of mathematical functions. New York: Dover.

Baatz, M. \& Wagner, G. 1997 Adaptive inertia caused by hidden pleiotropic effects. Theor. Popul. Biol. 51, 49-66.

Barton, N. 1990 Pleiotropic models of quantitative variation. Genetics 124, 773-782.
Barton, N. 1995 Linkage and the limits to natural selection. Genetics 140, 821-841.

Bulmer, M. G. 1973 The maintenance of the genetic variability of polygenic characters by heterozygous advantage. Genet. Res., Camb. 22, 9-12.

Carroll, L. 1965 Alice's adventures in Wonderland E Through the looking glass. New York: Airmont Publishing Company Inc. (First published 1872.).

Caspari, E. 1952 Pleiotropic gene action. Evolution 6, 1-18.

Clayton, G. A., Morris, J. A. \& Robertson, A. 1956 An experimental check on quantitative genetical theory. I. Short-term responses to selection. F. Genet. 55, 131-151.

Dobzhansky, T. \& Holz, A. M. 1943 A reexamination of the problem of manifold effects of genes in Drosophila melanogaster. Genetics 28, 295-303.

Falconer, D. S. \& MacKay, T. F. C. 1996 An introduction to quantitative genetics. Harlow: Longman.

Fisher, R.A. 1930 The genetical theory of natural selection. Oxford: Clarendon Press.

Gillespie, J. H. 1984 Pleiotropic overdominance and the maintenance of genetic variation in polygenic characters. Genetics $107,321-330$.

Griswold, C. K. \& Whitlock, M. C. 2003 The genetics of adaptation: the roles of pleiotropy, stabilizing selection and drift in shaping the distribution of bidirectional fixed mutational effects. Genetics 165, 2181-2192.

Haldane, J. B. S. 1927 A mathematical theory of natural and artificial selection part V. Selection and mutation. Proc. Camb. Phil. Soc. 23, 838-844.

Hill, W. G. \& Keightley, P. D. 1988 Interrelations of mutation, population size, artificial and natural selection. In Proceedings of the Second International Conference on Quantitative Genetics (ed. B. S. Weir, E. J. Eisen, M. M. Goodman \& G. Namkoong), pp. 57-70. Sunderland, MA: Sinauer.

Hill, W. G. \& Robertson, A. 1966 The effect of linkage on the limits to artificial selection. Genet. Res. Camb. 8, 269-294.

Keightley, P. D. 1994 The distribution of mutation effects on viability in Drosophila melanogaster. Genetics 138, 1315-1322.

Keightley, P. D. \& Hill, W. G. 1990 Variation maintained in quantitative traits with mutation-selection balance: pleiotropic side-effects on fitness traits. Proc. R. Soc. Lond. B 242, 95-100.

Keightley, P. D. \& Lynch, M. 2003 Toward a realistic model of mutations affecting fitness. Evolution 57, 683-685.

Kimura, M. 1983 The neutral theory of molecular evolution. Cambridge University Press.

Kondrashov, A. S. \& Turelli, M. 1992 Deleterious mutations, apparent stabilizing selection and the maintenance of quantitative variation. Genetics 132, 603-618.

Lande, R. 1980 The genetic covariance between characters maintained by pleiotropic mutations. Genetics 94, 203-215.

Lande, R. \& Arnold, S. J. 1983 The measurement of selection on correlated characters. Evolution 37, 1210-1226.

MacKay, T. F. C., Lyman, R. F. \& Jackson, M. S. 1992 Effects of $p$ element insertions on quantitative traits in Drosophila melanogaster. Genetics 130, 315-332.

Orr, H. A. 1998 The population genetics of adaptation: the distribution of factors fixed during adaptive evolution. Evolution 52, 935-949.

Orr, H. A. 1999 The evolutionary genetics of adaptation: a simulation study. Genet. Res. Camb. 74, 207-214.

Otto, S. P. \& Jones, C. D. 2000 Detecting the undetected: estimating the total number of loci underlying a quantitative trait. Genetics 156, 2093-2107.

Robertson, A. 1956 The effect of selection against extreme deviants based on deviation or on homozygosis. F. Genet. 54, 236-248. 
Slatkin, M. \& Frank, S. A. 1990 The quantitative genetic consequences of pleiotropy under stabilizing and directional selection. Genetics 125, 207-213.

Taylor, C. F. \& Higgs, P. G. 2000 A population genetics model for multiple quantitative traits exhibiting pleiotropy and epistasis. F. Theor. Biol. 203, 419-437.

Turelli, M. 1985 Effects of pleiotropy on predictions concerning mutation-selection balance for polygenic traits. Genetics 111, 165-195.

Wagner, G. P. 1989 Multivariate mutation-selection balance with constrained pleiotropic effects. Genetics 122, 223-234.
Wright, S. 1968 Evolution and the genetics of populations, vol. 1 (Genetics and biometric foundations). University of Chicago Press.

Wright, S. 1969 Evolution and the genetics of populations, vol. 2 (The theory of gene frequencies). University of Chicago Press. Zhang, X.-S. \& Hill, W. G. 2002 Pleiotropic model of maintenance of quantitative genetic variation at mutation-selection balance. Genetics 161, 419-433.

As this paper exceeds the maximum length normally permitted, the author has agreed to contribute to production costs. 